\title{
Three-dimensional Magnetic Properties of Soft Magnetic Composite Material at Different Frequencies
}

\author{
Yongjian Li, ${ }^{1,2}$ Zhi Wei Lin, ${ }^{2}$ Hongxun Liu, ${ }^{1}$ Yi Wang, ${ }^{2}$ Youguang Guo, ${ }^{2}$ \\ Jianguo Zhu, ${ }^{2}$ Qingxin Yang ${ }^{1,3}$ \\ ${ }^{1}$ Province-Ministry Joint Key Laboratory of Electromagnetic Field and Electrical Apparatus \\ Reliability, Hebei University of Technology, Tianjin, 300130, China \\ ${ }^{2}$ School of Electrical, Mechanical and Mechatronic Systems, University of Technology, \\ Sydney, PO Box 123, Broadway, NSW 2007, Australia \\ ${ }^{3}$ Electrical Engineering Department, Tianjin Polytechnic University, Tianjin, 300160, China
}


Due to the intrinsic isotropic magnetic properties, soft magnetic composite (SMC) materials are applied in electrical machines in which the magnetic energy can be transported in three dimensional (3-D). However, in real application, complicated magnetic properties, such as anisotropy and nonlinearity, are found, in particular, at the high frequency range. This paper studies the 3-D magnetic properties of SMC materials under complicated magnetization, such as circular, elliptical, and spherical excitations. The magnetic flux density vector $\mathbf{B}$ loci and magnetic field strength vector $\mathbf{H}$ loci and core losses at magnetization frequencies ranged from $50 \mathrm{~Hz}$ to $1000 \mathrm{~Hz}$ were measured and discussed using an improved 3-D testing system. Experimental results show that rotational core losses are greater than alternating losses at the same magnitude of flux density. In addition, rotational loss increases sharply after saturation point, but it is not observed in alternating loss. 


\section{INTRODUCTION}

Soft magnetic composite (SMC) materials produced by powder metallurgical techniques show some advantages: intrinsic magnetic and thermal isotropy; low eddy current loss and relatively low total core loss due to isolation layer and low manufacturing costs. ${ }^{1-3}$ The isotropic magnetic property bring crucial benefits for electromagnetic devices as the magnetic flux path in the devices can be three dimensional (3-D), and hence different topologies can be exploited to obtain high performances. ${ }^{4}$ Typical application of SMC materials are claw pole and transverse flux machines, in which 3-D magnetic flux density improve performance and simplify the machine structure. ${ }^{5,6}$ In recent years, 3-D magnetic properties of the SMC materials have been systematically investigated and modelled. ${ }^{7-10}$

However, in real electrical machines, complicated magnetic properties, such as anisotropy and nonlinearity, are found. In order to improve the performance of the SMC machine and open up new windows to apply this material in new devices, the 3D magnetic properties of this practical material must be understood in-depth. In this paper, the complicated vector B loci and corresponding $\mathbf{H}$ loci from $50 \mathrm{~Hz}$ to $1000 \mathrm{~Hz}$ are presented and discussed, and compared with alternating results

\section{EXPERIMENTAL DETAIL}

The existing 3-D magnetic property measurement system operated at low frequency was upgraded by employing adjustable excitation windings, as a result, the 3-D magnetic properties can be characterized up to $1000 \mathrm{~Hz}{ }^{11}$

The 3-D magnetic property tester mainly consists of three orthogonal square-frame yokes to guide the magnetic flux, and three pairs of adjustable excitation windings wrapped around the three pairs of orthogonal magnetic core poles used to produce magnetic fields along the three axes. The overall outline of the tester is a cubic with side length of $400 \mathrm{~mm}$. Yokes and cores are laminated structure with high performance grain-oriented material, as a result, relatively uniform and strong field of $1.9 \mathrm{~T}$ in the geometric centre of the tester can be generated. A $22 \times 22 \times 22 \mathrm{~mm}^{3}$ cubic SMC specimen, SOMALOYTM $500,{ }^{12}$ enclosed by $B-H$ sensing coils was mounted in the centre of the tester and slightly pressed by six core-poles. ${ }^{11}$ To ensure the measurement precision and diminish the systematic error, a $B-H$ sensing coil was designed to be small, thin and consistent. Comprehensive calibrations were carried out in a long solenoid and accurate coil coefficients of the sensing coils in three directions were obtained. The $\mathbf{H}$ vectors were detected by three pairs of $H$ sensing coils and the $\mathbf{B}$ vectors were measured by three pairs of small annular $B$ coils, which were embedded in the $H$ coils.

Various B loci in the specimen, such as circle, ellipse and sphere can be obtained by adjusting phase or frequency of sinusoidal excitation magnetic fields along three orthogonal directions. A spherical $\mathbf{B}$ loci and corresponding $\mathbf{H}$ loci, shown in Fig. 1, validates the 3-D tester working well. 


\section{RESULTS AND DISCUSSION}

Using updated 3-D magnetic properties tester, alternating and rotational 3-D magnetization experiments were carried out. Fig. 2 shows alternating core losses from $50 \mathrm{~Hz}$ to $1000 \mathrm{~Hz}$ along the $y$-axis. The core losses are consistent with the data from the manufacture. The alternating hysteresis loops and core losses measured in three directions are similar, but $y$-axis seems to be a easy magnetization direction. This is consistent with experiment data for rotational magnetization, shown in Fig. 3.

Fig. 3 illustrates a series of well-controlled elliptical B loci and corresponding $\mathbf{H}$ loci at $50 \mathrm{~Hz}$ in the xoy-, yoz- and zoxplane, in which the major axis of $\mathbf{B}$ is along $x$-, $y$-, and $z$-axis, respectively. The axis ratio $\varepsilon$, of the minor axis to the major axis (fixed at $1.6 \mathrm{~T}$ ) varies from 0 to 1. It can be seen that the plane of the $\mathbf{H}$ loci are parallel to the corresponding well-controlled plane of the $\mathbf{B}$ loci, in other words, $\mathbf{H}$ loci lie in the plane same as that of $\mathbf{B}$ loci. While $\mathbf{B}$ loci are well-controlled to be elliptical shape, the $\mathbf{H}$ loci are saddle-like shape. This seems to be caused by high order harmonics. comparing the most outer $\mathbf{H}$ loci in the xoy-plane with loci in the yoz- and zox-plane, it is found that $\mathbf{H}$ loci in the xoy-plane is square while the loci in the yoz- and zox-plane is rectangular. This slightly anisotropy is caused by compression of iron powder during SMC synthesis though the material is expected to be isotropy. Microscopic structure of the SMC materials shows that the interstice rate at the compression surface is higher than that at the side surface. ${ }^{13}$ Therefore, the particles are much closer and mass density is relatively high along the compaction direction. In this cubic SMC specimen, the compression direction is along the $y$-axis, which is so-called easy magnetization direction.

Figure 4 illustrates a series of well-controlled circular $\mathbf{B}$ loci in the xoy-plane and corresponding $\mathbf{H}$ loci in 3-D space at $200 \mathrm{~Hz}, 500 \mathrm{~Hz}$ and $1000 \mathrm{~Hz}$. As the magnetization frequency increases, the $\mathbf{H}$ loci, for example, in the xoy-plane, deflects from the corresponding B loci plane. The projections in the yoz- and zox-plane are not straight lines. Furthermore, the deflection degree increases with increasing excitation frequency. It is believed that such deflection is caused by coupling among core poles, but the projection components do not contribute to the core losses due to B loci in the in yoz- and zoxplanes are straight lines. Figure 5 shows rotational core losses as B loci were controlled to be circles in xoy-plane from $50 \mathrm{~Hz}$ to $1000 \mathrm{~Hz}$. The core losses at $50 \mathrm{~Hz}, 100 \mathrm{~Hz}, 200 \mathrm{~Hz}, 500 \mathrm{~Hz}$ and $1000 \mathrm{~Hz}$ are 3, 8, 27, 100, $220 \mathrm{~kW} / \mathrm{kg}$, respectively when the magnitude of circular $\mathbf{B}$ is 0.6 T. Compared with the alternating core losses shown in Fig. 2, the losses are 2, 5, 12, 42, 114 $\mathrm{W} / \mathrm{kg}$ at $0.6 \mathrm{~T}$. The rotational loss is much greater than that of alternating core loss, in particular, at higher frequencies. In addition, the rotational loss increases sharply above magnitude of $1 \mathrm{~T}$, however, this phenomenon does not observed in alternating loss. It is believed that the sharp increasing loss is attributed to the saturation point of $B=1 \mathrm{~T}$ from which the SMC material start saturation. 


\section{CONCLUSION}

Upgrading excitation windings in existing 3-D magnetic properties tester, 3-D magnetic hysteresis loci and core losses of soft magnetic composite material up to $1000 \mathrm{~Hz}$ were measured and discussed. The anisotropy introduced in SMC materials synthesis was observed in alternating and rotational magnetization in different planes. Rotational core losses are greater than alternating losses when magnitude of flux density is the same. In addition, rotational loss increases sharply after saturation point, but it is not observed in alternating loss. The deflection degree of plane of $\mathbf{H}$ loci from corresponding plane of $\mathbf{B}$ loci increases with increasing magnetization frequency due to coupling between core poles, but it does not contribute to the core losses. 


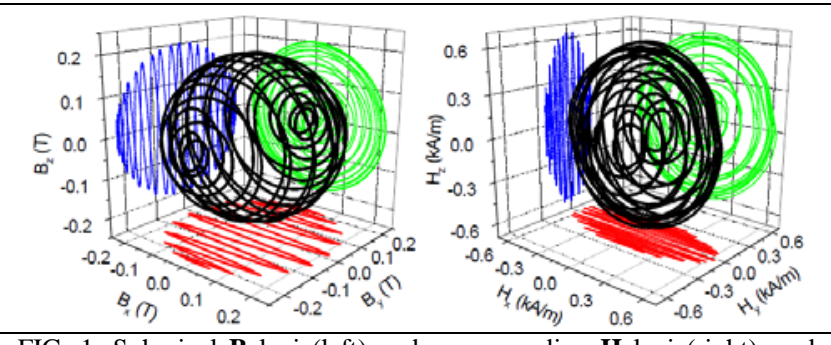

FIG. 1. Spherical $\mathbf{B}$ loci (left) and corresponding $\mathbf{H}$ loci (right), and projections in xoy-, yoz-, zox-planes. 


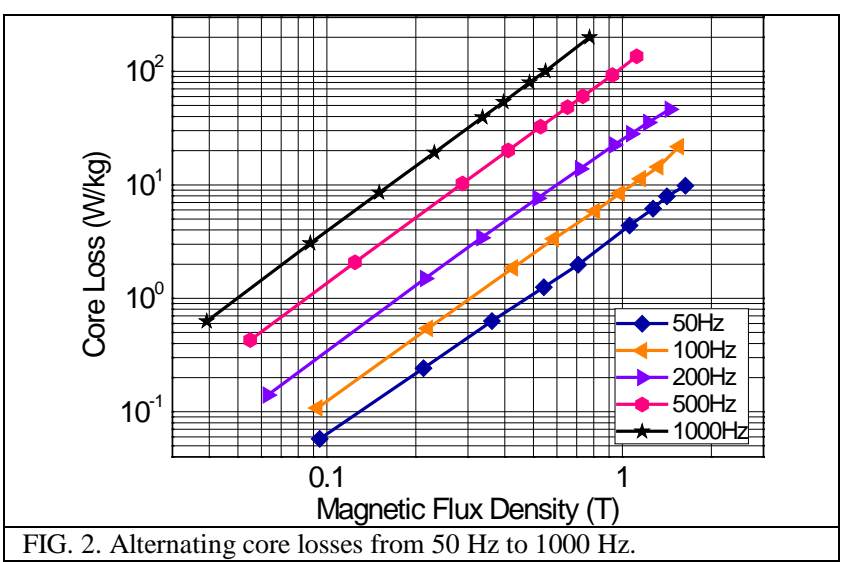




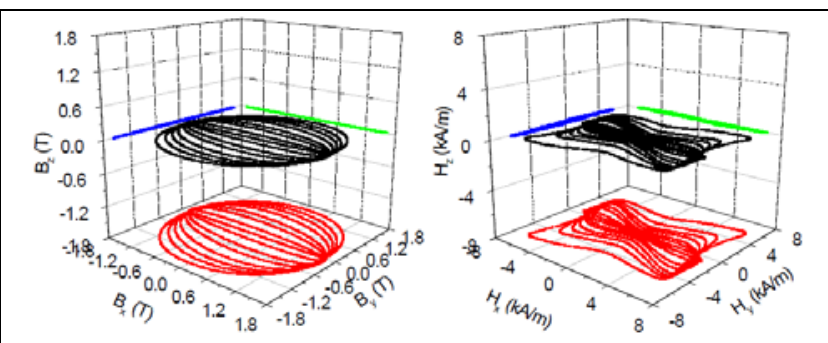

(a)
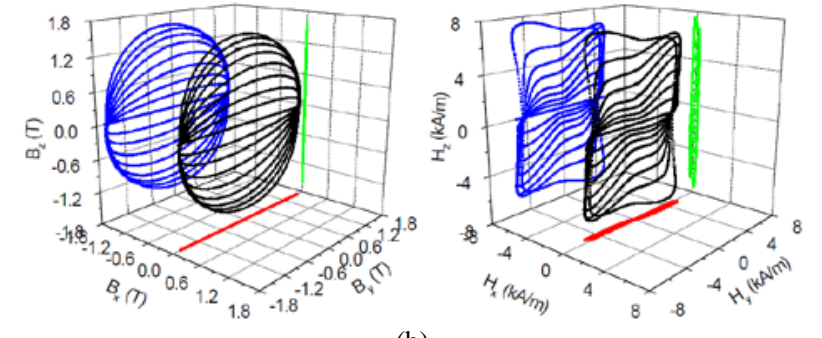

(b)
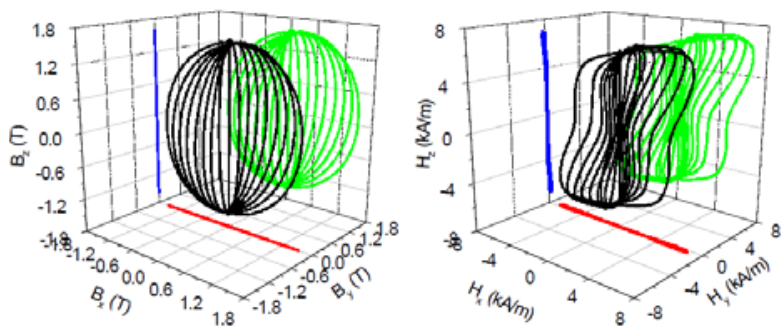

(c)

FIG. 3. Elliptical $\mathbf{B}$ loci ( $\varepsilon$ is from 0 to 1 ) (left), and corresponding $\mathbf{H}$ loci and projections in three planes at $50 \mathrm{~Hz}$ (right): (a) $\mathbf{B}, \mathbf{H}$ loci in the xoy-plane, the major axis is $x$; (b) $\mathbf{B}, \mathbf{H}$ loci in the yoz-plane, the major axis is $y$; (c) $\mathbf{B}, \mathbf{H}$ loci in the zox-plane, the major axis is $z$. 

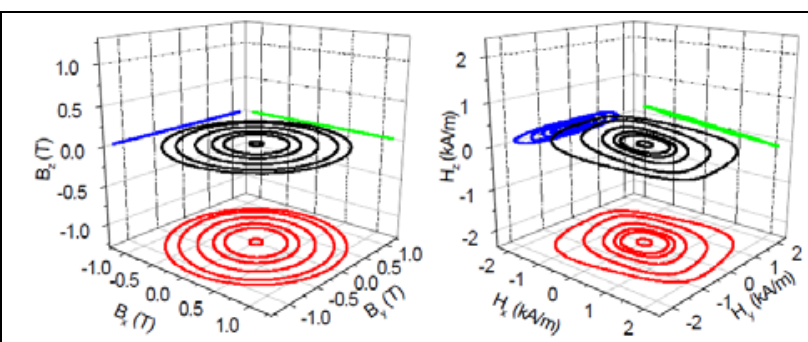

(a)
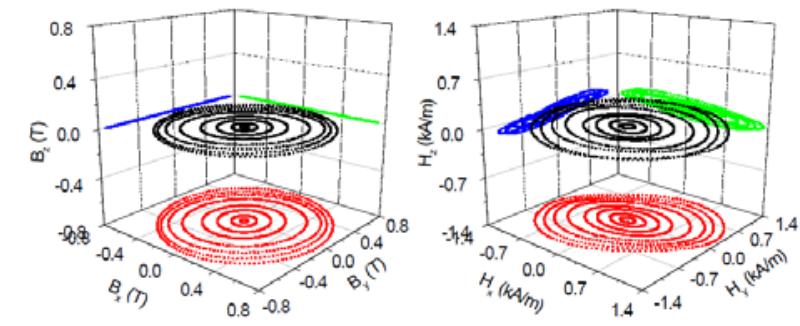

(b)
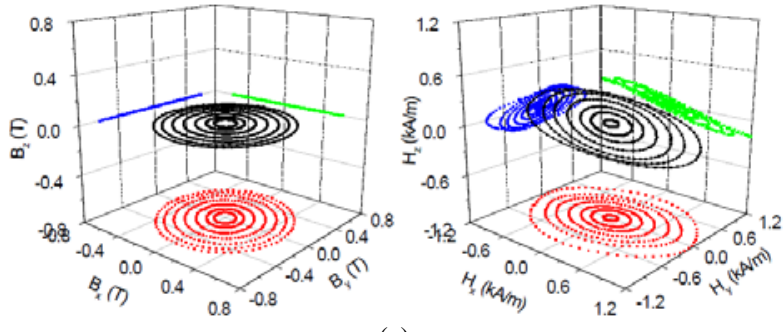

(c)

FIG. 4. Circular $\mathbf{B}$ loci in xoy-plane (left), and corresponding $\mathbf{H}$ loci (right): (a) $200 \mathrm{~Hz}$; (b) $500 \mathrm{~Hz}$; (c) $1000 \mathrm{~Hz}$ 


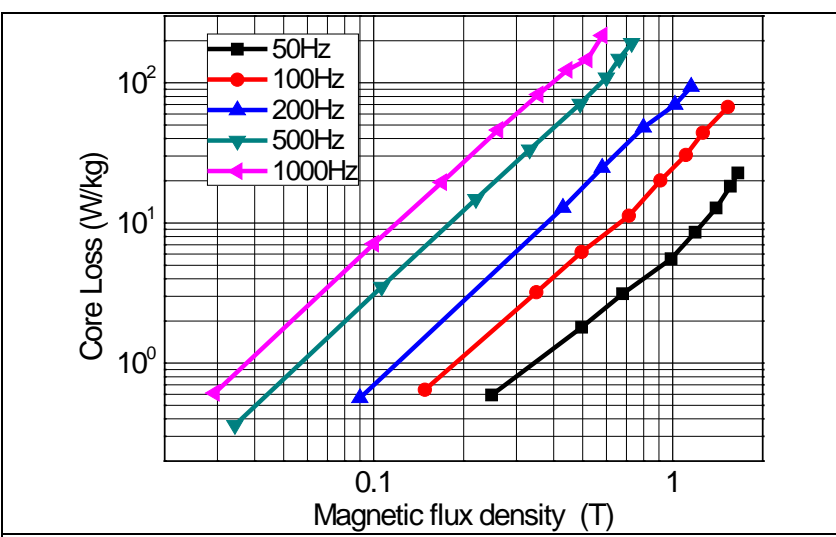

FIG. 5. Rotational core losses when circular $\mathbf{B}$ loci are controlled in the xoy-plane from $50 \mathrm{~Hz}$ to $1000 \mathrm{~Hz}$. 


\section{Reference}

${ }^{1}$ C. Cyr, P. Viarouge, S. Clenet, and J. Cros, IEEE Trans. Magn. 45, 1178 (2009).

${ }^{2}$ H. Shokrollahi, K. Janghorban, J. Mater. Process. Technol. 189, 1 (2007).

${ }^{3}$ Y. Cui, and G. B. Kliman, Industry Applications Conference (IAS). 546 (2004).

${ }^{4}$ Y. G. Guo, J. G. Zhu, P. A. Watterson and W. Wu, IEEE Trans. Ind. Appl. 39, 1696 (2003).

${ }^{5}$ Y. K Huang, J. G. Zhu, Y. G. Guo, Z. W. Lin, and Q. S. Hu, IEEE Trans. Magn. 43, 2492 (2007).

${ }^{6}$ Y. G. Guo, J. G. Zhu, and D. G. Dorrell, IEEE Trans. Magn. 45, 4582 (2009).

${ }^{7}$ J. G. Zhu, J. J. Zhong, Z. W. Lin, and J. D. Sievert, IEEE Trans. Magn. 39, 3429 (2003).

${ }^{8}$ J. J. Zhong, J. G. Zhu, Z. W. Lin, Y. G. Guo, and J. D. Sievert, J. Magn. Magn. Mater. 290, 1567 (2005).

${ }^{9}$ Z. W. Lin, H. W. Lu, J. G. Zhu, J. J. Zhong, X. L. Wang, and S. Y. Ding, J. Appl. Phys. 97, 10R306 (2005).

${ }^{10}$ Z. W. Lin, J. G. Zhu, and Y. G. Guo, J. Appl. Phys. 99, 08D909 (2006).

${ }^{11}$ Y. J. Li, J. G. Zhu, Q. X. Yang, Z. W. Lin, Y. G. Guo, and Y. Wang, IEEE Trans. Magn. 46, 1971 (2010).

${ }^{12}$ Reports of Höganäs AB, Sweden, 1997-2005, http:// www. Hoganas.com.

${ }^{13}$ Z. W. Lin and J. G. Zhu, J. Magn. Magn. Mater. 312, 158 (2007). 